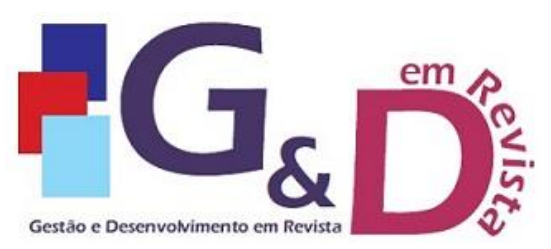

Gestão e Desenvolvimento em Revista V. 7, N. 1, jan-jun/2021, p. 59-70.

ISSN online: $2446-8738$

Artigo recebido em: 05/07/2021

Artigo aprovado em: 20/08/2021

\title{
CONTRIBUIÇÕES DA LÓGICA FUZZY À LOGÍSTICA: UMA REVISÃO SISTEMÁTICA DA LITERATURA
}

\author{
Nathan Peixoto Oliveira \\ Doutorado em Administração de Empresas pela Université de Bordeaux (França). Atualmente é \\ doutorando no Programa de Pós-graduacão em Engenharia de Produção pela Universidade Federal \\ de Santa Catarina - UFSC. \\ E-mail: nathanpeixot@yahoo.com.br \\ Rafaela Francisca Moreira Barbosa \\ Graduada em Engenharia de Produção. Atualmente é mestranda na Universidade Federal de Santa \\ Catarina - UFSC. \\ E-mail: barbosarafaela84@gmail.com
}

Carlos Manuel Taboada Rodriguez

Doutor em Engenharia Economica pela Technische Unversitat Dresden (1985). Atualmente é

Professor Titular no Departamento de Engenharia de Produção e Sistemas da Universidade Federal de Santa Catarina - UFSC.

E-mail: carlos.taboada@ufsc.br

\section{Resumo}

Para o aperfeiçoamento da logística, cada vez mais tem-se feito uso da lógica fuzzy com diversas aplicações, como por exemplo: controle de estoques, otimização de inventários, melhoria de processos logísticos, avaliação de fornecedores, geolocalização e seleção de parceiros. Diante desta tendência, este artigo teve como intuito fazer uma revisão sistemática utilizando a metodologia ProKnow- $C$ para analisar a difusão da lógica fuzzy, estratificando sua aplicação em 14 áreas distintas da logística. Como resultado, foram destacadas as principais contribuições da lógica fuzzy na logística internacional e no contexto brasileiro e oportunidades de pesquisa na área. Este trabalho além de delimitar o caminho da pesquisa realizado até o presente momento, tenta delinear possíveis perspectivas de estudo.

Palavras-chave: Lógica fuzzy; Logística; Cadeia de suprimentos.

\begin{abstract}
For the improvement of logistics, fuzzy logic has been increasingly used with various applications, such as: inventory control, inventory optimization, improvement of logistics processes, supplier evaluation, replacement and selection of partners. In view of this trend, this article aimed to carry out a systematic review using the ProKnow-C methodology to analyze the diffusion of fuzzy logic, stratifying its application in 14 distinct areas of logistics and research opportunities. As a result, they were highlighted as the main contributions of fuzzy logic in international logistics and in the Brazilian context. This work, in addition to delimiting the research path carried out so far, tries to outline possible study possibilities.
\end{abstract}

Keywords: Fuzzy logic; Logistics; Supply chain. 


\section{INTRODUÇÃO}

A logística é responsável pelo transporte e armazenagem de produtos, podendo facilitar o fluxo dos mesmos, desde o momento da aquisição das matérias primas até o consumo final (BALLOU, 2011). Para o referido autor a logística ainda busca criar um elo e valor entre os clientes e os fornecedores da empresa, além dos envolvidos indiretamente.

De acordo com Novaes (2015), a logística constitui de um processo composto por elementos que agregam valores de lugar, tempo, qualidade e informações para toda a cadeia produtiva, de modo que as necessidades dos clientes sejam atendidas. Empresas que gerenciam seus processos logísticos de forma eficiente, podem se destacar em um mercado altamente competitivo, fornecendo serviços de alto valor para seus clientes.

No ambiente logístico, considerar a competitividade como norte estratégico é tido como fundamental para um eficiente controle de custos (GRU, 2018). Um bom gerenciamento dos processos logísticos é de suma importância para que empresas possam controlar seus custos, e assim elevar o nível de sua competitividade (GEN et al., 2018).

Os processos logísticos se tornam cada vez mais desafiadores e importantes devido ao aumento da concorrência (SETHANAN; JAMRUS, 2020). Devido à complexidade do mercado e a intensiva competitividade, as tomadas de decisão devem ser bastante criteriosas. Algumas ferramentas são utilizadas para facilitar os processos decisórios nos diversos segmentos da logística, como exemplo a lógica fuzzy (JOVČıć et al., 2019).

A lógica fuzzy, surgiu no ano de 1930, pelo filósofo Jan Lukasiewicz, a partir de estudos relacionados a termos como, alto, quente, velho, onde propôs a utilização de um intervalo de valores [0,1] (MARRO et al., 2010). Ainda segundo os autores, em 1965, Lofti Zadech publicou o artigo Fuzzy Sets que se consolidou posteriormente como a origem da lógica fuzzy.

A lógica fuzzy é uma ferramenta bastante difundida nos diversos campos científicos, pois tornou-se uma solução de problemas complexos para estes, devido à semelhança com o raciocínio humano e o auxílio nas tomadas de decisão (HERGHILIGIU et al., 2019). De acordo com Kumar (2019), formaliza e estrutura o raciocínio, e possui traços de raciocínio parecido com os dos humanos, além de auxiliar o processo de tomada de decisão (FERDAUS et al., 2020).

Diante desse contexto apresentado, o objetivo deste trabalho está em identificar os principais segmentos de aplicação da lógica fuzzy no campo da logística por meio de um panorama das publicações no setor. A importância de revisões sistemáticas de lógica fuzzy no campo logístico poderá auxiliar, tanto no âmbito acadêmico quanto no empresarial, diversos tomadores de decisão. Além disso, a ferramenta possui ampla aplicação em segmentos logísticos como logística verde, reversa e sustentável, logística humanitária, portuária, entre outros.

Para alcançar o objetivo proposto, este trabalho está estruturado em quatro tópicos principais: introdução, método, resultados e conclusões. O primeiro tópico apresentou uma breve contextualização a respeito dos temas principais do trabalho. No tópico dois, será mostrado o passo a passo realizado para a obtenção da amostra de artigos, como por exemplo as palavras-chave utilizadas na pesquisa e os 
critérios de seleção de artigos. No tópico de resultados, será mostrado os principais resultados alcançados. E por fim no tópico 4, a conclusão.

\section{REFERENCIAL TEÓRICO}

\subsection{LÓGICA FUZZY}

A lógica fuzzyé, portanto, um método heurístico que utiliza a teoria de conjuntos fuzzy, integrando a área de inteligência artificial, no campo das aplicações da Ciência Cognitiva, juntamente com os algoritmos genéticos e as redes neurais (O'BRIEN, 2001). Foi desenvolvida a partir de $1965 \mathrm{com}$ os trabalhos do professor Lotfi Zadeh, para representar o conhecimento incerto ou impreciso (SANDRI; CORREA, 1999).

Segundo Heizer e Render (2001), a lógica fuzzy permite trabalhar com valores aproximados, influências e dados incompletos ou ambíguos para tomar decisões. Além disso, a lógica fuzzy pode ser comparada com a lógica clássica (ou booleana), que trata com a definição clássica de conjuntos. Nessa definição, um certo valor pertence ou não a um determinado conjunto, sendo representado pelos valores 0 e 1 , respectivamente. No caso da lógica fuzzy, um certo valor pode pertencer a um determinado conjunto com um certo grau de pertinência (SANTOS et al., 2012).

O desenvolvimento de um trabalho com a lógica fuzzy pode ser resumido em três etapas, de acordo com Nobre (2000) e Santos et al., (2012). A primeira etapa consiste em propor um conjunto de regras linguísticas sendo acrescentados pelos conectivos lógicos "and" e "or. Uma variável linguística é definida como uma entidade utilizada para representar de modo impreciso e, por tanto, linguístico, um conceito ou uma variável de um determinado problema, admitindo como valores apenas expressões linguísticas como "baixo", "alto", médio", entre outros de acordo com a necessidade de exemplificar o sistema (LANDMANN; ERDMANN, 2011).

O segundo passo consiste em definir as funções de pertinência que devem caracterizar de modo quantitativo os valores das variáveis linguísticas. Com isso, o terceiro e último passo consiste em definir inferências para manipulação das regras e para a tomada de decisão dos envolvidos no modelo, definindo interfaces de entrada e saída do método. A interface de entrada possui como objetivo mapear as informações numéricas do ambiente estudado para valores caracterizados por funções de pertinência, transformando informações quantitativas em qualitativas (fuzzyfication) e a interface de saída o oposto (defuzzyfication) (LANDMANN; ERDMANN, 2011; GANGA et al., 2011; SANTOS et al., 2012).

O objetivo da lógica fuzzy é estudar os princípios do raciocínio aproximado, fazendo com que as decisões tomadas por um sistema computacional se aproximem cada vez mais das decisões humanas (CAMARGOS, 2002).

\section{MÉTODO}

O presente trabalho é caracterizado como uma pesquisa descritiva, tendo em vista o objetivo do estudo. Na pesquisa descritiva, cabe ao pesquisador realizar o estudo, a análise, o registro e a interpretação dos dados buscados, além de descrever as características de uma população, experiência ou fenômeno (GIL, 2008). Com isso, foi realizado uma revisão sistemática da literatura utilizando o método ProKnow-C (Knowledge Development Process - Constructivist), criado pelo 
laboratório MCDA - Multi-criteria Decision Analysis - da Universidade Federal de Santa Catarina. O modelo prevê a realização de três etapas:

a) Seleção de artigos brutos: escolher dos eixos de pesquisa "lógica fuzzy" e "logística"; definir as palavras-chave e operadores booleanos; escolher o banco de dados (Web of Science e Scopus); definir os limitantes de pesquisa (periódicos, sem limitação temporal, pesquisa em "título, resumo e palavras-chave" dos artigos); verificar alinhamento com o tema de pesquisa;

b) Filtragem do banco de artigos: importar banco de artigos bruto para um software de revisão bibliográfica (EndNote); excluir artigos em duplicidade e publicados em congressos ou livros; realizar a leitura dos títulos pertinentes à pesquisa excluindo os desalinhados; exportar para uma planilha e ordenar artigos por ordem de citação, assim como sua porcentagem individual do total e acumulada; realizar o corte de representatividade para determinar os artigos com reconhecimento científico comprovado realizando a leitura do resumo destes e determinação do banco de autores; reanalisar os artigos sem reconhecimento científico permitindo uma repescagem baseada no banco de autores, tempo de publicação e leitura de resumo; realizar a leitura integral dos artigos finalistas;

c) Teste de representatividade do portfólio bibliográfico primário: analisar as referências secundárias dos artigos do portfólio bibliográfico primário com as mesmas limitações do banco de artigo bruto; delimitar as referências secundárias alinhadas ao tema de pesquisa pelo título; ordenar artigos por ordem de citação (baseado no google acadêmico), sua porcentagem individual do total e acumulada; realizar 0 corte de representatividade igual ao do banco de artigos bruto (por número de citação ou por percentual); para os artigos com representatividade, analisar se há reconhecimento científico, caso não, realizar uma análise pela leitura do resumo e do artigo; juntar os artigos do portfólio primário e do teste de representatividade.

Considerando a seleção de artigos brutos, nos critérios de inclusão de artigos foram considerados somente artigos científicos em versão final e idiomas em inglês e português. Após essa triagem inicial, os artigos que no seu resumo, título ou palavras-chave não consideravam logística e lógica fuzzy não foram incluídos para análise. 


\section{RESULTADOS}

\subsection{SELEÇÃO DE ARTIGOS BRUTOS}

Para compor a primeira triagem (bruta) de artigos, foram delimitados dois eixos de pesquisa. O primeiro eixo, "logística", diz respeito a área principal de estudo, já o segundo, "lógica fuzzy", refere-se a ferramenta ou método utilizado para solucionar problemas na área já citada. Para a escolha das palavras-chave, para o eixo "logística", utilizou-se "logistic ${ }^{\star "}$, enquanto, para o eixo "lógica fuzzy", utilizou-se de "Fuzzy Logic", "Fuzzy Number", "Fuzzy Set" ou "Fuzzification", conforme pode ser visto na Figura 1 a seguir:

Figura 1 - Eixos e palavras-chave

Eixo 1 - Logística

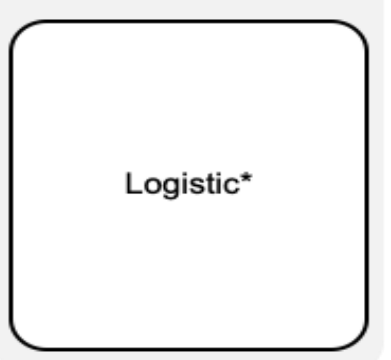

\section{Eixo 2 - Lógica Fuzzy}

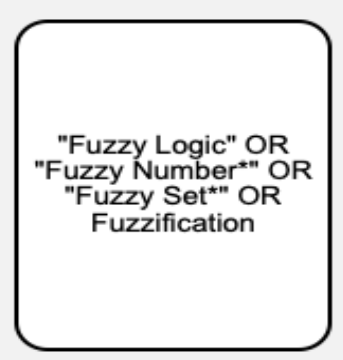

Fonte: Autores (2021)

Fez-se a busca em duas das principais bases de dados: Web of Science e Scopus. E, como limitantes definiu-se: para tempo, um intervalo desde a criação das bases até a data de 20 de dezembro de 2020.; para local de pesquisa, resultados encontrados nos títulos, resumo e palavras-chave dos artigos; para tipo de documento, restringiu-se a pesquisa de artigos e artigos de revisão. Como resultado da busca na base Scopus, foram encontrados 745 artigos, já na base Web of Science, 1.278, totalizando 2.023 artigos. Para definir o alinhamento com o tema de pesquisa, foram selecionados 5 artigos por ordem de citação de cada base, chegando-se aos seguintes artigos, conforme Tabela 1. A partir da mesma e da leitura dos artigos foi possível validar a pertinência dos mesmos quanto ao tema de pesquisa. 


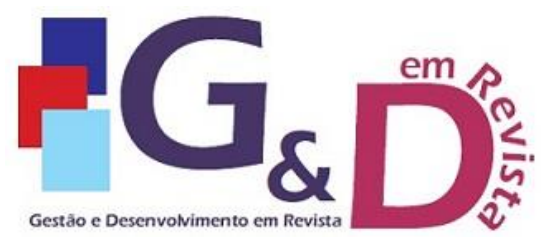

Gestão e Desenvolvimento em Revista V. 7, N. 1, jan-jun/2021, p. 59-70.

ISSN online: $2446-8738$

Artigo recebido em: 05/07/2021

Artigo aprovado em: 20/08/2021

Tabela 1 - Análise de alinhamento ao tema de pesquisa

\begin{tabular}{|c|c|c|c|}
\hline Autores & Título & Citações & BBase \\
\hline West, D. & Neural network credit scoring models & 577 & SScopus \\
\hline Chen, C.T. & $\begin{array}{l}\text { A fuzzy approach to select the location of the } \\
\text { distribution center }\end{array}$ & 338 & SScopus \\
\hline $\begin{array}{l}\text { Yalcin A., Reis S., Aydinoglu } \\
\text { A.C., Yomralioglu T. }\end{array}$ & $\begin{array}{l}\text { A GIS-based comparative study of frequency ratio, } \\
\text { analytical hierarchy process, bivariate statistics and } \\
\text { logistics regression methods for landslide susceptibility } \\
\text { mapping in Trabzon, NE Turkey }\end{array}$ & 337 & SScopus \\
\hline $\begin{array}{l}\text { Chang B., Chang C.-W., Wu } \\
\text { C.-H. }\end{array}$ & $\begin{array}{l}\text { Fuzzy DEMATEL method for developing supplier } \\
\text { selection criteria }\end{array}$ & 310 & SScopus \\
\hline $\begin{array}{l}\text { Lu L.Y.Y., Wu C.H., Kuo T.- } \\
\text { C. }\end{array}$ & $\begin{array}{l}\text { Environmental principles applicable to green supplier } \\
\text { evaluation by using multi-objective decision analysis }\end{array}$ & 257 & SScopus \\
\hline $\begin{array}{l}\text { Pourghasemi, Hamid Reza; } \\
\text { Pradhan, Biswajeet; } \\
\text { Gokceoglu, Candan }\end{array}$ & $\begin{array}{l}\text { Application of fuzzy logic and analytical hierarchy } \\
\text { process (AHP) to landslide susceptibility mapping at } \\
\text { Haraz watershed, Iran }\end{array}$ & 405 & SScopus \\
\hline $\begin{array}{l}\text { Kannan, Devika; } \\
\text { Khodaverdi, Roohollah; } \\
\text { Olfat, Laya; Jafarian, } \\
\text { Ahmad; Diabat, Ali }\end{array}$ & $\begin{array}{l}\text { Integrated fuzzy multi criteria decision making method } \\
\text { and multi-objective programming approach for supplier } \\
\text { selection and order allocation in a green supply chain }\end{array}$ & 312 & SScopus \\
\hline $\begin{array}{l}\text { Kannan, Devika; Lopes de } \\
\text { Sousa Jabbour, Ana } \\
\text { Beatriz; Chiappetta Jabbour, } \\
\text { Charbel Jose }\end{array}$ & $\begin{array}{l}\text { Selecting green suppliers based on GSCM practices: } \\
\text { Using fuzzy TOPSIS applied to a Brazilian electronics } \\
\text { company }\end{array}$ & 311 & SScopus \\
\hline $\begin{array}{l}\text { Devkota, Krishna Chandra; } \\
\text { Regmi, Amar Deep; } \\
\text { Pourghasemi, Hamid Reza; } \\
\text { Yoshida, Kohki; Pradhan, } \\
\text { Biswajeet; Ryu, In Chang; } \\
\text { Dhital, Megh Raj; } \\
\text { Althuwaynee, Omar F. }\end{array}$ & $\begin{array}{l}\text { Landslide susceptibility mapping using certainty factor, } \\
\text { index of entropy and logistic regression models in GIS } \\
\text { and their comparison at Mugling-Narayanghat road } \\
\text { section in Nepal Himalaya }\end{array}$ & 310 & SScopus \\
\hline $\begin{array}{l}\text { Kannan, Govindan; } \\
\text { Pokharel, Shaligram; } \\
\text { Kumar, P. Sasi }\end{array}$ & $\begin{array}{l}\text { A hybrid approach using ISM and fuzzy TOPSIS for the } \\
\text { selection of reverse logistics provider }\end{array}$ & 282 & SScopus \\
\hline
\end{tabular}

Fonte: Autores (2021)

\subsection{FILTRAGEM DO BANCO DE ARTIGOS}

Após extraídos os resultados das bases para o software EndNote, um conjunto de dados oriundos da Scopus e Web of Science foi criado e, para evitar a duplicidade de informações, foram excluídos os artigos presentes em ambas as bases. Foram encontrados então 433 valores repetidos, culminando em 1.590 artigos exclusivos.

Em seguida, foi feita a leitura dos títulos a fim de se encontrar artigos desalinhados com o objetivo de pesquisa, resultando em 119 artigos excluídos e uma base posterior de 1.471 artigos. Esta foi exportada para o software Excel $\AA$, ordenados por citação, bem como a participação percentual (absoluta e acumulada) 


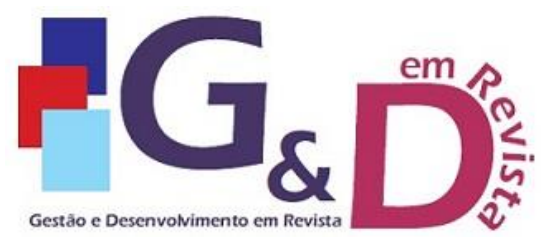

Gestão e Desenvolvimento em Revista V. 7, N. 1, jan-jun/2021, p. 59-70.

ISSN online: $2446-8738$

Artigo recebido em: 05/07/2021

Artigo aprovado em: 20/08/2021

da citação de cada artigo do total de citações da base, conforme pode ser visto na Figura 2.

Figura 2 - Fragmento da extração para o Excel

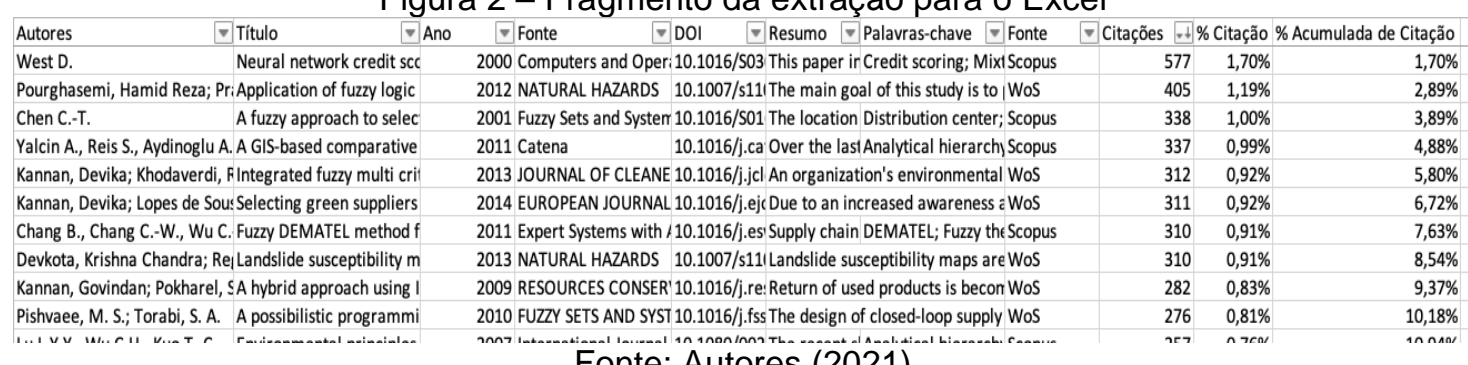
Fonte: Autores (2021)

O corte dos dados do Excel foi realizado quando o percentual acumulado de citações chegou a $80 \%$. Desta forma 356 artigos $(24,20 \%$ da base) representava cerca de $80 \%$ do total de citações, conforme pode ser visto na Figura 3.

Figura 3 - Corte do percentual acumulado de citações

\begin{tabular}{|c|c|c|c|c|}
\hline 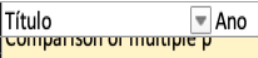 & 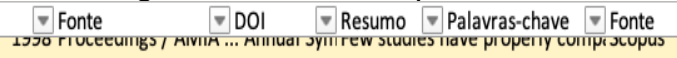 & $\nabla$ Citações & $\frac{+4 \% \text { Citação }}{2.0}$ & $\begin{array}{r}\text { \% Acumulada de Citação } \\
\qquad 1,4 / 10\end{array}$ \\
\hline Evaluating military trainiı & 2015 Aerospace Science ar 10.1016/j.asi The combina AHPTOPSIS; Fuzzy Ic Scopus & & $25 \quad 0,07 \%$ & $79,54 \%$ \\
\hline A Fuzzy Reverse Logistics & 2016 International Journal 10.1007/s40: This paper di Economic order/prc Scopus & & $0,07 \%$ & $79,62 \%$ \\
\hline Analysing the interaction & 2018 INTERNATIONAL JOU 10.1080/002 Emergencies and disasters place WoS & & $0,07 \%$ & $79,69 \%$ \\
\hline Location optimization of $\mathrm{r}$ & 2012 Journal of Zhejiang U 10.1631/jzus Locating dist Axiomatic fuzzy set Scopus & & $0,07 \%$ & $79,76 \%$ \\
\hline Application of analytic hit & 2016 ENVIRONMENTAL EA 10.1007/s12। The purpose of the present study WoS & & $0,07 \%$ & $79,84 \%$ \\
\hline An interactive possibilistiı & 2017 APPLIED SOFT COMP 10.1016/j.asi This paper presents a new multi-WoS & & $0,07 \%$ & $79,91 \%$ \\
\hline Decision model for count। & 2006 International Journal $10.1007 / s 00$ Clothing maı Clothing manufactu Scopus & & $0,07 \%$ & $79,98 \%$ \\
\hline Multicriteria fuzzy classifi & 2004 Fuzzy Sets and Systern 10.1016/S01 In this paper Astrocytic tumour; I Scopus & & $0,07 \%$ & $80,05 \%$ \\
\hline Application of frequency I & 2015 ARABIAN JOURNAL 0 10.1007/s12: The main goal of this study was t WoS & & $0,07 \%$ & $80,12 \%$ \\
\hline A dynamic multi-plant lot & 2016 INTERNATIONAL JOU 10.1080/002 In this paper, we investigate a m WoS & & $0,07 \%$ & $80,19 \%$ \\
\hline An Integrated Hybrid App & 2020 JOURNAL OF CLEANE 10.1016/j.jcl In recent decades, reverse logist WoS & & $0,07 \%$ & $80,26 \%$ \\
\hline A new method for manag & 2012 Annals of Onerations $10.1007 / \mathrm{s} 10$. The internati Hierarchical multi-t Sconus & & $0.07 \%$ & $80.33 \%$ \\
\hline
\end{tabular}
Fonte: Autores (2021)

\subsection{TESTE DE REPRESENTATIVIDADE PORTFÓLIO PRIMÁRIO}

Os artigos em amarelo (cerca de $80 \%$ do percentual acumulado de citações conforme Figura 3) são os dito artigos com reconhecimento científico. Assim, os demais (sem reconhecimento da comunidade científica), são reanalisados tendo como critérios o pertencimento dos seus autores ao banco de autores dos artigos com reconhecimento científico, ao tempo de publicação (dando preferência para artigos publicados nos últimos 5 anos) e leitura do resumo.

Nesta etapa todos os artigos sem reconhecimento da comunidade científica foram considerados na repescagem e voltaram a compor o banco de artigos com reconhecimento científico. Pela suficiência de artigos, não foi necessário a realização do teste de representatividade (que iria buscar referências secundárias no portfólio bibliográfico primário). Foram obtidos então 1.471 artigos que representam o uso da Lógica Fuzzy na área de Logística.

\subsection{APLICAÇÕES DA LÓGICA FUZZYNA LOGÍSTICA}

Baseado no portfólio bibliográfico, foram levantados os principais usos da lógica fuzzy por área da logística, conforme Quadro 1 a seguir. 
Quadro 1 - Principais aplicações da lógica fuzzy por área da logística

\begin{tabular}{|c|c|}
\hline Área da Logística & Aplicação da Lógica Fuzzy \\
\hline $\begin{array}{l}\text { Logística Verde, Reversa e } \\
\text { Sustentável }\end{array}$ & Geolocalização e Centros de Distribuição, Fábricas, \\
\hline $\begin{array}{l}\text { Logística Hospitalar } \\
\text { Logística Humanitária }\end{array}$ & $\begin{array}{l}\text { Avaliação interna: qualidade do atendimento, turnover, } \\
\text { clima organizacional. }\end{array}$ \\
\hline Logística Lean & $\begin{array}{l}\text { Nível de eficiência, desperdícios, desempenho dos } \\
\text { funcionários. }\end{array}$ \\
\hline Logística Urbana & $\begin{array}{l}\text { Avaliação da malha, dos veículos e maquinário, da } \\
\text { necessidade de manutenção }\end{array}$ \\
\hline Logística Portuária & $\begin{array}{l}\text { Avaliação de fornecedores: preço, excelência, } \\
\text { experiência }\end{array}$ \\
\hline $\begin{array}{l}\text { Operador Logístico e Lead } \\
\text { Logistic Provider }\end{array}$ & $\begin{array}{l}\text { Seleção de parceiros: confiabilidade, capilaridade, } \\
\text { integridade }\end{array}$ \\
\hline Interoperabilidade & $\begin{array}{l}\text { Avaliação da interoperabilidade de peças, softwares, } \\
\text { produção (joint-venture). }\end{array}$ \\
\hline $\begin{array}{l}\text { Construindo o conceito de } \\
\text { Logística do Varejo }\end{array}$ & $\begin{array}{l}\text { Atendimento aos clientes: nível de satisfação, avaliação } \\
\text { dos produtos, avaliação do SAC. }\end{array}$ \\
\hline Logística 4.0 & $\begin{array}{l}\text { Grau de inovação: número de patentes, nível de } \\
\text { eficiência dos projetos de inovação, ranking da empresa dentre as } \\
\text { mais inovadoras. }\end{array}$ \\
\hline $\begin{array}{l}\text { Big Data, Inteligência } \\
\text { Artificial e Machine Learning na } \\
\text { Logística }\end{array}$ & $\begin{array}{l}\text { Registro do histórico de informações para construção do } \\
\text { triângulo fuzzy e auxílio decisório }\end{array}$ \\
\hline $\begin{array}{l}\text { Sistema de Equações } \\
\text { Estruturais na Logística }\end{array}$ & $\begin{array}{l}\text { Análise Multicritério: conexão entre } 2 \text { ou mais constructos } \\
\text { (conjuntos fuzzy) e a relação entre eles }\end{array}$ \\
\hline Blockchain na Logística & $\begin{array}{l}\text { Avaliação da rede integrada de parceiros: nível } \\
\text { confiança, segurança das informações, número de parceiros }\end{array}$ \\
\hline Riscos na Logística & $\begin{array}{l}\text { Riscos de ruptura (falta de produtos), capacidade } \\
\text { produtiva e estocagem. }\end{array}$ \\
\hline
\end{tabular}

Fonte: Autores (2021) 


\subsection{APLICAÇÕES DA LÓGICA FUZZY NO CONTEXTO BRASILEIRO}

Com base no Portfólio Bibliográfico, 47 artigos tiveram aplicações práticas no contexto brasileiro e apresentaram enfoque em:

a) (23\%) Instalações logísticas: posicionamento de CDs, fábricas e do varejo;

b) (41\%) Apoio a decisão logística: avaliação externa (seleção de fornecedores, parceiros, terceiros) e avaliação interna (atendimento ao cliente, desempenho dos funcionários, adequação à filosofia Lean, avaliação dos processos logísticos);

c) (36\%) Sustentabilidade: riscos geológicos (deslizamento de encostas) aliado a problemas de transporte, gestão ambiental (logística reversa e economia circular) e

d) (menor que 1\%) Existe uma quantidade pouco significativa na área biomédica com interseção logística.

\section{CONCLUSÕES}

O presente estudo teve como objetivo identificar os principais segmentos de aplicação da lógica fuzzy no campo da logística por meio de um panorama das publicações no setor. Face aos resultados encontrados, é possível verificar a ampla utilização da ferramenta nas 14 áreas da logística listada nos trabalhos.

Além disso, no contexto brasileiro, o uso da lógica fuzzy na logística está concentrado em publicações para solução de problemas de instalações logísticas (23\%), ferramenta de apoio a decisão logística $(41 \%)$, sustentabilidade $(36 \%)$ e de modo pouco significante (menor que $1 \%$ ) a problemas da biomedicina vinculados a logística. Foram apresentados também os principais autores de ambas as bases de pesquisa - Scopus e Web of Science - (Tabela 1) e na seção seguinte serão elencadas as oportunidades de pesquisa encontradas na literatura.

De acordo com o estudo realizado, observou-se lacunas do conhecimento em 3 áreas e que podem ser consideradas como oportunidades de pesquisa de logística com a aplicação da ferreamente lógica fuzzy:

a) Logística Hospitalar: análise da variação de insumos hospitalares (como oxigênio, bolsas de sangue, medicamentos etc.) para definição do ponto de pedido via lógica fuzzy e criação de gatilhos automáticos de ordem de compra pelos hospitais.

b) Logística Urbana e Big Data / Machine Learning: geolocalização do ponto ótimo para sinalização urbana (placas, semáforos, faixas de circulação) para leitura e interpretação de carros autônomos. 
c) Gestão de Riscos e Machine Learning: avaliação de indicadores do modal aeroviário para pilotagem automática, como pressão atmosférica, altitude, longitude, índice pluviométrico, umidade, velocidade, aceleração, fluxo de aeronaves, origem e destino.

A lógica fuzzy aplicada ao contexto logístico tem como principal contribuição o correto direcionamento para os tomadores de decisão, pois a lógica computacional se aproxima do pensamento racional humano. A combinação da lógica fuzzy com a expertise dos stakeholders no processo de decisão de problemas complexos pode possuir grande valor prático, contribuindo também para o embasamento teórico e a difusão de mais pesquisas na área.

\section{Referências}

BALLOU, R. H. Logística empresarial: transporte, administração de materiais e distribuição física. 24⿳亠丷a ed. São Paulo: Atlas, 2011.

CAMARGOS, F. L. Lógica Nebulosa: uma abordagem filosófica e aplicada. Florianopolis: UFSC, 2002.

CHANG, B.; CHANG, C.; WU, C. Fuzzy DEMATEL method for developing supplier selection criteria. Expert systems with Applications, v. 38, n. 3, p. 1850-1858, 2011. Disponível em: <https://doi.org/10.1016/j.eswa.2010.07.114>.

CHEN, C. A fuzzy approach to select the location of the distribution center. Fuzzy sets and systems, v. 118, n. 1, p. 65-73, 2001. Disponível em: < https://doi.org/10.1016/S0165-0114(98)00459-X>.

DEVKOTA, K.; REGMI, A.; POURGHASEMI, H.; YOSHIDA, K. Landslide susceptibility mapping using certainty factor, index of entropy and logistic regression models in GIS and their comparison at Mugling-Narayanghat road section in Nepal Himalaya. Natural hazards, v. 65 , n. 1, p. 135-165, 2013. Disponível em: https://doi.org./10.1007/s11069-012-0347-6>.

GANGA, G. M.; CARPINETTI, C. R.; POLITANO, P. R. Gestão do desempenho em cadeias de suprimentos usando lógica fuzzy $A$ fuzzy logic approach to supply chain performance management. Gestão e Produção, São Carlos, v. 18, n. 4, p. 755-774, 2011.

GIL, A. C. Métodos e técnicas de pesquisa social. 6ª Ed. Rio de Janeiro: Atlas, 2008.

GEN, M.; LIN, L.; YUN, Y. Recent advances in hybrid priority-based genetic algorithms for logistics and SCM network design. Computers and Industrial Engineering, v. 125, n. September, p. 394-412, 2018. Disponível em: $<$ https://doi.org/10.1016/j.cie.2018.08.025>.

GRU, V. Minimizing the trade-off between sustainability and cost-effective performance by using autonomous vehicles., Journal of Cleaner Production. v. 184, p. 709-717, 2018. Disponível em: < https://doi.org/10.1016/j.jclepro.2018.02.302>. 


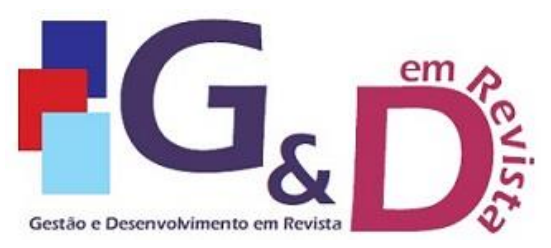

Gestão e Desenvolvimento em Revista V. 7, N. 1, jan-jun/2021, p. 59-70.

ISSN online: $2446-8738$

Artigo recebido em: 05/07/2021

Artigo aprovado em: 20/08/2021

HERGHILIGIU, I.; ROBU, I.; VILCU, A.; ASANDULUI, A.; AVASILCAI, S.; BALAN, C. Sustainable environmental management system integration and business performance: A balance assessment approach using fuzzy logic. Sustainability (Switzerland), v. 11, n. 19, 2019. Disponível em: < https://doi.org/10.3390/su11195311>.

HEIZER, J.; RENDER, B. Administração de operações 5. ed. Rio de Janeiro: Livros Técnicos e Científicos, 2001.

JOVČIĆ, S.; PRUSA, P.; DOBRODOLAC, M.; SVADLENKA, L. A proposal for a decision-making tool in third-party logistics (3PL) provider selection based on multicriteria analysis and the fuzzy approach. Sustainability (Switzerland), v. 11, n. 15, 2019. Disponível em: < https://doi.org/10.3390/su11154236>

KANNAN, D.; KHODAVERDI, R.; OLFAT, L.; JAFARIAN, A. Integrated fuzzy multi criteria decision making method and multi-objective programming approach for supplier selection and order allocation in a green supply chain. Journal of Cleaner production, $\quad$ v. 47, p. 355-367, 2013. Disponível em: <https://doi.org/0.1016/j.jclepro.2013.02.010>.

KANNAN, D.; DE SOUSA J.; JABBOUR, C. Selecting green suppliers based on GSCM practices: Using fuzzy TOPSIS applied to a Brazilian electronics company. European Journal of Operational Research, v. 233, n. 2, p. 432-447, 2014. Disponível em: < http://dx.doi.org/10.1016/j.ejor.2013.07.023>.

KANNAN, G.; POKHAREL, S.; KUMAR, P. A hybrid approach using ISM and fuzzy TOPSIS for the selection of reverse logistics provider. Resources, conservation and recycling, v. 54, n. 1, p. 28-36, 2009. Disponível em: <http://doi.org/10.1016/j.resconrec.2009.06.004>.

KUMAR, A.; SHARMA, A. Systematic literature review of fuzzy logic-based text summarization. Iranian Journal of Fuzzy Systems, v. 16, n. 5, p. 45-59, 2019. Disponível em: <http://doi.org/10.22111//JFS.2019.4906>.

LU, Y.; WU, C. H.; KUO, T.-C. Environmental principles applicable to green supplier evaluation by using multi-objective decision analysis. International journal of production research, v. 45, n. 18-19, p. 4317-4331, 2007. Disponível em: < https://doi.org/10.1080/00207540701472694>.

MARRO, A. A; MATHEUS, A.; SOUZA, C. Lógica fuzzy: conceitos e aplicações. Natal: Universidade Federal do Rio Grande do Norte (UFRN), 2010.

NOVAES, A. Logística e gerenciamento da cadeia de distribuição. Rio de Janeiro: Elsevier, 2015.

O'BRIEN, J. A. Sistemas de informação e as decisões gerenciais na era da internet São Paulo: Saraiva, 2001.

POURGHASEMI, H. R.; PRADHAN, B.; GOKCEOGLU, C. Application of fuzzy logic and analytical hierarchy process (AHP) to landslide susceptibility mapping at Haraz watershed, Iran. Natural hazards, v. 63, n. 2, p. 965-996, 2012. Disponível em: < https://doi.org/10.1007/s11069-012-0217-2>.

SANTOS, A. V. N.; FELIX, L.B.; VIEIRA, J. G. V. Estudo da logística de distribuição 
física de um laticínio utilizando lógica fuzzy. Revista Produção, v. 22, n. 3, p. 576583, 2012. Disponível em: < http://dx.doi.org/10.1590/S0103-65132012005000036>.

SANDRI, S.; CORREA, C. Lógica Nebulosa. São José dos Campos: ITA, 1999.

SETHANAN, K.; JAMRUS, T. Hybrid differential evolution algorithm and genetic operator for multi-trip vehicle routing problem with backhauls and heterogeneous fleet in the beverage logistics industry. Computers and Industrial Engineering, v. 146, n. May, p. 106571, 2020. Disponível em: $<$ https://doi.org/10.1016/j.cie.2020.106571>.

WEST, D. Neural network credit scoring models. Computers \& Operations Research, v. 27, n. 11-12, p. 1131-1152, 2000. Disponível: < https://doi.org/10.1016/S0305-0548(99)00149-5>.

YALCIN, A. A GIS-based comparative study of frequency ratio, analytical hierarchy process, bivariate statistics and logistics regression methods for landslide susceptibility mapping in Trabzon, NE Turkey. Catena, v. 85, n. 3, p. 274-287, 2011. Disponível em: http://doi.org/10.1016/j.catena.2011.01.014>.

ZADEH, L. A. Fuzzy sets. Information and control, v. 8, n. 3, p. 338-353, 1965. Disponível em: < https://doi.org/10.1016/S0019-9958(65)90241-X>. 